\title{
Approximate Solutions and Symmetry Group for Initial-Value Problem of Nonlinear Cahn-Hilliard Equation
}

\author{
Jina $\mathrm{Li}^{1, *}$, Hong $\mathrm{Li}^{1}$, Suli $\mathrm{Zuo}^{2}$ and Cong $\mathrm{Gu}^{1}$ \\ ${ }^{I}$ College of Science, Zhongyuan University of Technology, Zhengzhou 450007, China \\ ${ }^{2}$ School of Mathematics, Northwest University, Xi'an 710069, China
}

\begin{abstract}
In this paper, for the nonlinear Cahn-Hilliard equation, we give its symmetry group by the approximate generalized conditional symmetry. As the application of approximate generalized conditional symmetry, the initial-value problem of the partial differential equations can be reduced to perturbed initial-value problem for a system of perturbed first-order ordinary differential equations. By solving the reduced ordinary differential equations, we obtain the approximate solutions of the initial-value problem of research equations. At the last, some exaples be given to show the reduction procedure.
\end{abstract}

Keywords: Approximate solution, symmetry group, symmetry reduction.

\section{INTRODUCTION}

There are many nonlinear partial differential equations (PDEs) with small parameters or perturbed equations arising from the real world, so it is of great importance and interest to find approximate solutions and extend the scope and depth of the perturbation theory $[1,2]$. There are ordinary methods for studying the approximate solutions of perturbed equations by the perturbation methods in combination with the Lie group theory [3]. Recently, several symmetry based perturbation methods have been developed to deal with the perturbation properties of perturbed equations [4-8]. Actually, these methods are effective ways investigating perturbed PDEs [9]. In ref. [10-12], the authors successfully handle with the initial-value problem by the generalized conditional symmetry (GCS) which was introduced by Fushchych and Zhdanov [13], and independently by Fokas and Liu [14]. In ref. [15], we have solved the approximate symmetry reduction for initial-value problems of the extended KdV-Burgers equations with perturbation.

In this paper, we intend to study the initial value problem of the nonlinear Cahn-Hilliard equation [16] with perturbation

$u_{t}=-\left(F(u) u_{x}\right)_{x}-\varepsilon u_{x x x x}$,

$\alpha(x ; \varepsilon) u_{x}\left(t_{0}, x\right)+\beta(x ; \varepsilon) u\left(t_{0}, x\right)=\gamma(x ; \varepsilon)$.

Here $t, x$ are two independent variables and $u$ is a scalar dependent one. The Cahn-Hilliard equation was propounded by Cahn and Hilliard in 1958 as a mathematical model which describes the diffusion phenomena in phase transition. Then the equation can characterize the process in the context of the continuum theory of phase transitions, the nonlinearity $\mathrm{F}(\mathrm{u})$ is the derivative of a double-well potential with wells of equal depth and $0 \prec \varepsilon \prec 1$ shows the thickness of an interface separating the two preferred states of the system. Later, many mathematicians considered the Cahn-Hilliard type equation and have done lots of remarkable results $[17,18]$, such as the perturbation of solutions [19], the existence, stability and uniqueness of solutions [20], etc. So this paper mainly researches the initial-value problem of the Cahn-Hilliard equation by approximate generalized conditional symmetry (AGCS).

If we treat the perturbed PDE

$\eta\left(t, x, u, u_{1} \ldots, u_{n} ; \varepsilon\right)=O\left(\varepsilon^{2}\right)$

as an Nth-order ordinary differential equations (ODEs) with respect to variable $x$, where $u_{1}=\frac{\partial u}{\partial x}, u_{2}=\frac{\partial^{2} u}{\partial x^{2}}, \cdots$, then its general integral can be expressed (locally) in the form

$u(t, x ; \varepsilon)=U\left(t, x, \phi_{1}(t), \phi_{2}(t), \ldots, \phi_{n}(t) ; \varepsilon\right)$,

where $\phi_{j}(t),(j=1, \ldots, N)$ are arbitrary smooth functions.

In order to integrate Eq. (3), it would be natural to consider higher-order AGCSs which are linear in the variables $u, u_{1}, \ldots u_{n}$ of the form

$Q=\sum_{k=o}^{\infty}\left[D_{x}^{k}\left(u_{N}-\sum_{i=o}^{N-1}\left(a_{i}(t, x)+\varepsilon b_{i}(t, x)\right) u_{i}\right)\right] \frac{\partial}{\partial u_{k}}$,

$u_{0}=u$. 
After the AGCSs are found, Eq. (3) can be integrated to an ansatz of the following form

$u=\sum_{i=1}^{n} \Omega_{i}(t, x ; \varepsilon) \phi_{i}(t)$.

\section{MAIN RESULTS}

In what follows, the cases $N=2,3.4 .5$ of AGCSs (5) are studied, and all the possible inequivalent forms of Eq. (1) which admit AGCSs (5) are described. Here we show the classification results of Eq. (1) admitting second, third-, fourth- and fifth-order AGCSs (5) as follows:

Theorem 1. Eq. (1) admits the second-order AGCSs of the form

$Q=\eta \frac{\partial}{\partial u} \equiv\left[u_{2}-\sum_{i=0}^{1}\left(a_{i}(t, x)+\varepsilon b_{i}(t, x)\right) u_{i}\right] \frac{\partial}{\partial u}, u_{0}=u$.

if and only if it is equivalent to one of the following ones:

(i) $u_{t}=-\left(\left(f_{1} u^{2}+f_{2} u+f_{3}\right) u_{x}\right)_{x}-\varepsilon u_{x x x x}$,

$\eta=u_{x x}$

(ii) $u_{t}=-\left(\left(f_{1} u+f_{2}\right) u_{x}\right)_{x}-\varepsilon u_{x x x x}$,

$\eta=u_{x x}-\varepsilon b_{1} u_{x}$

$$
u_{t}=-f_{1} u_{x x}-\varepsilon u_{x x x x} \text {, }
$$

(iii) $\eta=u_{x x}-\left(a_{0}(t, x)+\varepsilon b_{0}(t, x)\right) u-\left(a_{1}(t, x)\right.$

$$
\left.+\varepsilon b_{1}(t, x)\right) u \text {. }
$$

Here $f_{1}, f_{2}, f_{3}$ are arbitrary constants and functions $a_{i}(t, x), b_{i}(t, x)(i=0,1)$ satisfy the following systems of PDEs respectively.

$$
\begin{aligned}
a_{0 t} & =-2 f_{1} a_{1 x} a_{0}-f_{1} a_{0 x x}, \\
a_{1 t} & =-f_{1} a_{1 x x}-2 f_{1} a_{0 x}-2 f_{1} a_{1} a_{1 x}, \\
b_{0 t} & =-6 a_{1} a_{1 x x} a_{0}-6 a_{0} a_{0 x x}-8 a_{1 x}^{2} a_{0}-6 a_{1 x x} a_{0 x} \\
& -a_{0 x x x}-4 a_{0 x}^{2}-f_{1} b_{0 x x}-4 a_{1 x} a_{0 x x}-4 a_{1 x x x} a_{0} \\
& -2 f_{1} a_{1 x} b_{0}-4 a_{0} a_{1} a_{0 x}-4 a_{1 x} a_{0}^{2}-4 a_{1}^{2} a_{1 x} a_{0} \\
& -4 a_{1} a_{1 x} a_{0 x}-2 f_{1} b_{1 x} a_{0}, \\
b_{1 t}= & -4 a_{1} a_{1 x x x}-4 a_{1}^{3} a_{1 x}-2 f_{1} b_{0 x}-4 a_{0 x x x} \\
& -10 a_{1 x} a_{1 x x}-12 a_{1} a_{1 x}^{2}-4 a_{0 x} a_{1}-6 a_{0} a_{1 x x} \\
& -a_{1 x x x x}-4 a_{0} a_{0 x}-6 a_{1}^{2} a_{1 x x}-6 a_{0 x x} a_{1}-8 a_{0} a_{1} a_{1 x} \\
& -2 f_{1} a_{1} b_{1 x}-12 a_{0 x} a_{1 x}-2 f_{1} a_{1 x} b_{1}-f_{1} b_{1 x x} .
\end{aligned}
$$

In the following, we will give the computational procedure.

According to the approximate generalized conditional symmetry, we obtain the determining equation

$$
F_{5} \varepsilon^{5}+F_{4} \varepsilon^{4}+F_{3} \varepsilon^{3}+F_{2} \varepsilon^{2}+F_{1} \varepsilon^{1}+F_{0}=O\left(\varepsilon^{2}\right)
$$

Eq. (11) stands for infinitesimals of the same order for $\varepsilon^{2}$, so we omit the value of $F_{2}, F_{3}, F_{4}, F_{5}$. Here

$$
\begin{aligned}
F_{0}= & -F_{u u u} u_{x}^{4}-5 a_{1}(t, x) F_{u u} u_{x}^{3}-\left[3 a_{0}(t, x) F_{u}\right. \\
& +4 a_{1}(t, x)^{2} F_{u}+4 a_{1 x}(t, x) F_{u} \\
& \left.+6 a_{0}(t, x) F_{u u} u\right] u_{x}^{2}-\left[\left(7 a_{0}(t, x) a_{1}(t, x)\right.\right. \\
& \left.\left.+4 a_{0 x}(t, x)\right) F_{u} u\right]+\left(a_{1 x x}(t, x)+2 a_{0 x}(t, x)\right. \\
& \left.\left.+2 a_{1}(t, x) a_{1 x}(t, x)\right) F_{u}+a_{1 t}(t, x)\right] u_{x} \\
& -\left[a_{0 t}(t, x)-a_{0 x x}(t, x) F\right. \\
& \left.-2 a_{1 x}(t, x) a_{0}(t, x) F\right] u-3 a_{0}(t, x)^{2} F_{u} u^{2},
\end{aligned}
$$$$
F_{1}=-5 b_{1}(t, x) F_{u u} u_{x}^{3}-\left[6 b_{0}(t, x) u F_{u u}+\left(3 b_{0}(t, x)\right.\right.
$$$$
\left.\left.+4 b_{1 x}(t, x)+8 a_{1}(t, x) b_{1}(t, x)\right) F_{u}\right] u_{x}^{2}
$$$$
-\left[\left(7 a_{0}(t, x) b_{1}(t, x)+7 b_{0}(t, x) a_{1}(t, x)\right.\right.
$$$$
\left.+4 b_{0 x}(t, x)\right) F_{u} u+10 a_{1 x}(t, x) a_{1 x x}(t, x)
$$$$
+8 a_{0}(t, x) a_{1}(t, x) a_{1 x}(t, x)
$$$$
+12 a_{0 x}(t, x) a_{1 x}(t, x)+6 a_{1}(t, x)^{2} a_{1 x x}(t, x)
$$$$
+4 a_{0}(t, x) a_{0 x}(t, x)+2 F b_{0 x}(t, x)
$$$$
+4 a_{0 x}(t, x) a_{1}(t, x)^{2}+12 a_{1}(t, x) a_{1}(t, x)
$$$$
+2 F b_{1 x}(t, x) a_{1}(t, x)+b_{1 t}(t, x)
$$$$
+4 a_{1}(t, x) a_{1 x x x}(t, x)+F b_{1 x x}(t, x)
$$$$
+6 a_{1}(t, x) a_{0 x x}(t, x)+4 a_{1 x}(t, x) a_{1}(t, x)^{3}
$$$$
+6 a_{0}(t, x) a_{1 x x}(t, x)+2 F a_{1 x}(t, x) b_{1}(t, x)
$$$$
\left.+4 a_{0 x x x}(t, x)+a_{1 x x x x}(t, x)\right] u_{x}
$$$$
-6 a_{0}(t, x) b_{0}(t, x) f_{u} u^{2}-\left[4 a_{0 x}(t, x)^{2}\right.
$$$$
+F b_{0 x x}(t, x)+4 a_{1 x}(t, x) a_{0 x x}(t, x)
$$$$
+4 a_{0}(t, x) a_{1}(t, x) a_{0 x}(t, x)+2 F a_{0}(t, x) b_{1 x}(t, x)
$$$$
+b_{0 t}(t, x)+4 a_{0}(t, x) a_{1 x}(t, x) a_{1}(t, x)^{2}
$$$$
+4 a_{1 x}(t, x) a_{1}(t, x) a_{0 x}(t, x)
$$$$
+6 a_{0}(t, x) a_{1}(t, x) a_{1 x x}(t, x)+2 F a_{1 x}(t, x) b_{0}(t, x)
$$$$
+4 a_{0}(t, x) a_{1 x x x}(t, x)+4 a_{1 x}(t, x) a_{0}(t, x)^{2}
$$$$
+a_{0 x x x x}(t, x)+8 a_{0}(t, x) a_{1 x}(t, x)^{2}
$$$$
\left.+6 F a_{0}(t, x) a_{0 x x}(t, x)+6 a_{1 x x}(t, x) a_{0 x}(t, x)\right] u,
$$

$F_{0}, F_{1}$ are polynomials of the derivative of $u$. 
Set

$F_{0}=0$,

$F_{1}=0$.

In above equations, the coefficients of the derivative of $u$ are equal to zero, so the theorem 1 is proved.

Theorem 2. Eq. (1) admits the third-order AGCSs of the form

$Q=\eta \frac{\partial}{\partial u} \equiv\left[u_{3}-\sum_{i=0}^{2}\left(a_{i}(t, x)+\varepsilon b_{i}(t, x)\right) u_{i}\right] \frac{\partial}{\partial u}$,

$u_{0}=u$.

if and only if it is equivalent to one of the following ones:

(i) $u_{t}=-\left(\left(f_{1} u+f_{2}\right) u_{x}\right)_{x}-\varepsilon u_{x x x x}$,

$$
\eta=u_{x x x}
$$

(ii) $u_{t}=-f_{1} u_{x x}-\varepsilon u_{x x x x}$,

$$
\begin{aligned}
\eta= & u_{x x x}-\left[a_{2}(t, x)+\varepsilon b_{2}(t, x)\right] u_{x x} \\
& -\left[a_{1}(t, x)+\varepsilon b_{1}(t, x)\right] u_{x} \\
& -\left[a_{0}(t, x)+\varepsilon b_{0}(t, x)\right] u .
\end{aligned}
$$

Here functions $a_{i}(t, x), b_{i}(t, x)(i=0,1,2) \quad$ satisfy the following systems of PDEs respectively.

$$
\begin{aligned}
a_{0 t}= & -f_{1} a_{0 x x}-2 f_{1} a_{0} a_{2 x}, \\
a_{1 t}= & -f_{1} a_{1 x x}-2 f_{1} a_{1} a_{2 x}-2 f_{1} a_{0 x}, \\
a_{2 t}= & -f_{1} a_{2 x x}-2 f_{1} a_{2} a_{2 x}, \\
b_{0 t}= & -4 a_{0} a_{1} a_{2 x}-6 a_{0} a_{2} a_{2 x x}-4 a_{0} a_{2} a_{1 x} \\
& -4 a_{2} a_{0 x} a_{2 x}-2 f_{0} b_{0} a_{2 x}-8 a_{0} a_{2 x}^{2} \\
& -2 f_{1} a_{0} b_{2 x}-4 a_{0} a_{2 x x x}-4 a_{0} a_{0 x} \\
& -6 a_{0} a_{1 x x}-4 a_{0} a_{2}^{2} a_{2 x}-f_{1} b_{0 x x} \\
& -6 a_{0 x} a_{2 x x}-4 a_{2}^{2} a_{2 x} a_{0}-f_{1} b_{x x} \\
& -6 a_{0 x} a_{2 x x}-4 a_{0 x} a_{1 x}-4 a_{2 x} a_{0 x x} \\
& -a_{0 x x x x}, \\
= & -2 f_{1} b_{1} a_{2 x}-4 a_{1} a_{2 x} a_{2}^{2}-6 a_{1} a_{1 x x} \\
& -6 a_{1} a_{2} a_{2 x x}-4 a_{2} a_{1 x} a_{2 x}-4 a_{1} a_{2} a_{1 x} \\
& -4 a_{1}^{2} a_{2 x}-4 a_{0} a_{1 x}-4 a_{1} a_{0 x}-8 a_{0 x} a_{2 x} \\
& -2 f_{1} b_{0 x}-6 a_{1 x} a_{2 x x}-2 f_{1} a_{1} b_{2 x}-f_{1} b_{1 x x} \\
& -4 a_{1} a_{2 x x x}-6 a_{0} a_{2 x x}-4 a_{0} a_{2} a_{2 x} \\
& -8 a_{1} a_{2 x}^{2}-4 a_{1 x}^{2}-4 a_{0 x x x}-a_{1 x x x x} \\
& -4 a_{2 x} a_{1 x x},
\end{aligned}
$$

$$
\begin{aligned}
b_{2 t}= & -12 a_{1 x} a_{2 x}-2 f_{1} b_{1 x}-6 a_{1} a_{2 x x}-6 a_{2} a_{1 x x} \\
& -4 a_{1} a_{1 x}-4 a_{1 x} a_{2}^{2}-4 a_{2} a_{2 x x x}-2 f_{1} b_{2} a_{2 x} \\
& -4 a_{2} a_{0 x}-4 a_{0} a_{2 x}-6 a_{2}^{2} a_{2 x x}-2 f_{1} a_{2} b_{2 x} \\
& -12 a_{2} a_{2 x}^{2}-8 a_{1} a_{2} a_{2 x}-6 a_{0 x x}-f_{1} b_{2 x x} \\
& -10 a_{2 x} a_{2 x x}-4 a_{1 x x x}-4 a_{2}^{3} a_{2 x}-a_{2 x x x x} .
\end{aligned}
$$

The computational procedure of Theorem 2 is similar to Theorem 1, so we omit it.

When Eq. (1) admits the fourth- and fifth-order AGCSs, the linear equations can be obtained, so we leave out the two cases. The following examples show the reduction procedure.

Example 1. Approximate reduction of Eq. (7) to Cauchy problem.

Integrating Eq. (8) yields the ansatz $u(t, x ; \varepsilon)$

$u(t, x ; \varepsilon)=\phi_{1}(t) x+\phi_{2}(t)$.

Firstly, by calculating the approximate Lie symmetry of $\eta=u_{x x}$ in the following equation,

$$
\begin{aligned}
X= & \left(\zeta_{1}(t, x)+\varepsilon \zeta_{2}(t, x)\right) \frac{\partial}{\partial x}+\left[\left(m_{1}(t, x)\right.\right. \\
& \left.\left.+\varepsilon m_{1}(t, x)\right) u+n_{1}(t, x)+\varepsilon n_{1}(t, x)\right] \frac{\partial}{\partial u}
\end{aligned}
$$

so we obtain the following equation

$$
\begin{aligned}
& {\left[m_{2 x x} u+n_{2 x x}+\left(2 m_{2 x}-\zeta_{2 x x}\right) u_{x}\right] \varepsilon} \\
& +m_{1 x x} u+n_{1 x x}+\left(2 m_{1 x}+\zeta_{1 x x}\right) u_{x}=O\left(\varepsilon^{2}\right) .
\end{aligned}
$$

Set

$$
\begin{aligned}
& m_{2 x x}=0, \\
& n_{2 x x}=0, \\
& 2 m_{2 x}-\zeta_{2 x x}=0, \\
& m_{1 x x}=0, \\
& n_{1 x x}=0, \\
& 2 m_{1 x}-\zeta_{1 x x}=0 .
\end{aligned}
$$

Solving above equations, we obtain

$$
\begin{aligned}
& m_{1}=h_{7}(t) x+h_{8}(t), \\
& m_{2}=h_{5}(t) x+h_{6}(t), \\
& n_{1}=h_{3}(t) x+h_{4}(t), \\
& n_{2}=h_{1}(t) x+h_{2}(t), \\
& \zeta_{1}=h_{7}(t) x^{2}+h_{11}(t) x+h_{12}(t),
\end{aligned}
$$


$\zeta_{2}=h_{5}(t) x^{2}+h_{9}(t) x+h_{10}(t)$,

Set

$$
\begin{aligned}
& \alpha(x ; \varepsilon)=\zeta_{1}\left(t_{0}, x\right)+\varepsilon \zeta_{2}\left(t_{0}, x\right), \\
& \beta(x ; \varepsilon)=-\left(m_{1}\left(t_{0}, x\right)+\varepsilon m_{2}\left(t_{0}, x\right)\right), \\
& \gamma(x ; \in)=n_{1}\left(t_{0}, x\right)+\varepsilon n_{2}\left(t_{0}, x\right) .
\end{aligned}
$$

Here $h_{i}\left(t_{0}\right)=C_{i}$ and yields the perturbed initial-value conditions for Eq. (7)

$\left[C_{7} x^{2}+C_{11} x+C_{12}+\varepsilon\left(C_{5} x^{2}+C_{9} x+C_{10}\right)\right] u_{x}\left(t_{0}, x\right)$

$-\left[C_{7} x+C_{8}+\varepsilon\left(C_{5} x+C_{6}\right)\right] u\left(t_{0}, x\right)$

$=C_{3} x+C_{4}+\varepsilon\left(C_{1} x+C_{2}\right)$.

By inserting (16) into the initial-value problem (7) and (17), we have the following Cauchy problem:

$\frac{d \phi_{1}}{d t}=-2 f_{1} \phi_{1}(t)^{3}$

$\frac{d \phi_{2}}{d t}=-\left(f_{2}+2 f_{1} \phi_{2}(t)\right) \phi_{1}(t)^{2}$,

$\phi_{1}\left(t_{0}\right)=A_{1}+\varepsilon A_{2}+O\left(\varepsilon^{2}\right)$,

$\phi_{2}\left(t_{0}\right)=B_{1}+\varepsilon B_{2}+O\left(\varepsilon^{2}\right)$.

$A_{i}, B_{i}(i=1,2)$ are the arbitrary constants which are related to $C_{i}(i=1, \ldots, 12)$.

$$
\begin{aligned}
A_{1}= & \frac{C_{7} C_{4}-C_{3} C_{8}}{C_{8}^{2}-C_{11} C_{8}+C_{7} C_{12}}, \\
A_{2}= & \frac{C_{7} C_{2}+C_{4} C_{5}-C_{3} C_{6}-C_{1} C_{8}}{C_{8}^{2}-C_{11} C_{8}+C_{7} C_{12}} \\
& +\frac{C_{3} C_{8}-C_{4} C_{7}}{\left(C_{8}^{2}-C_{11} C_{8}+C_{7} C_{12}\right)^{2}} \\
& +\frac{2 C_{6} C_{8}-C_{8} C_{9}+C_{5} C_{12}-C_{6} C_{11}+C_{7} C_{19}}{\left(C_{8}^{2}-C_{11} C_{8}+C_{7} C_{12}\right)^{2}}, \\
B_{1}= & \frac{C_{4} C_{11}-C_{3} C_{12}-C_{4} C_{8}}{C_{8}^{2}-C_{11} C_{8}+C_{7} C_{12}}, \\
B_{2}= & \frac{C_{2} C_{11}+C_{4} C_{9}-C_{4} C_{6}-C_{1} C_{12}-C_{2} C_{8}-C_{3} C_{10}}{C_{8}^{2}-C_{11} C_{8}+C_{7} C_{12}} \\
& +\frac{C_{3} C_{12}-C_{4} C_{11}+C_{4} C_{8}}{\left(C_{8}^{2}-C_{11} C_{8}+C_{7} C_{12}\right)^{2}} \\
& +\frac{2 C_{6} C_{8}-C_{8} C_{9}+C_{5} C_{12}-C_{6} C_{11}+C_{7} C_{10}}{\left(C_{8}^{2}-C_{11} C_{8}+C_{7} C_{12}\right)^{2}} .
\end{aligned}
$$

The following two approximate solutions are given by solving the Eqs. (18)
$\phi_{1}(t)=-\frac{1}{M(t)}$,

$\phi_{2}(t)=-\frac{f_{2}}{2 f_{1}}-\frac{f_{2}+2 f_{1} B_{1}+2 \varepsilon f_{1} B_{2}}{2 f_{1}\left(A_{1}+\varepsilon A_{2}\right) M(t)}$,

and

$\phi_{1}(t)=\frac{1}{M(t)}$,

$\phi_{2}(t)=-\frac{f_{2}}{2 f_{1}}+\frac{f_{2}+2 f_{1} B_{1}+2 \varepsilon f_{1} B_{2}}{2 f_{1}\left(A_{1}+\varepsilon A_{2}\right) M(t)}$,

where $M(t)=\sqrt{4 f_{1} t-\frac{K}{\left(A_{1}+\varepsilon A_{2}\right)^{2}}}$

$$
K=4 f_{1} t_{0} A_{1}^{2}+8 \varepsilon f_{1} t_{0} A_{1} A_{2}+4 \varepsilon^{2} f_{1} t_{0} A_{2}^{2}-1 .
$$

Then the two approximate solutions are obtained by substituting the above expressions for functions $\phi_{1}(t), \phi_{2}(t)$ into (16).

Example 2. Approximate reduction of Eq. (14) to Cauchy problem.

Integrating Eq. (15) yields the ansatz $u(t, x ; \in)$

$$
u(t, x ; \varepsilon)=\phi_{1}(t) x^{2}+\phi_{2}(t) x+\phi_{3}(t)(19)
$$

Applying the above algorithm, we can get the perturbed initial-value conditions for Eq. (14)

$$
\begin{aligned}
& {\left[\frac{1}{2} C_{9} x^{2}+C_{13} x+C_{14}+\varepsilon\left(\frac{1}{2} C_{7} x^{2}+C_{11} x\right.\right.} \\
& \left.\left.+C_{12}\right)\right] u_{x}\left(t_{0}, x\right)-\left[C_{9} x+C_{10}+\varepsilon\left(C_{7} x+C_{8}\right)\right] u\left(t_{0}, x\right) \\
& =\frac{1}{2} C_{4} x^{2}+C_{5} x+C_{6}+\varepsilon\left(\frac{1}{2} C_{1} x^{2}+C_{2} x+C_{3}\right),
\end{aligned}
$$

By inserting (19) into the initial-value problem (14) and (20), we have the following Cauchy problem:

$\frac{d \phi_{1}}{d t}=-6 f_{1} \phi_{1}(t)^{2}$,

$$
\begin{aligned}
& \frac{d \phi_{2}}{d t}=-6 f_{1} \phi_{2}(t) \phi_{1}(t), \\
& \frac{d \phi_{3}}{d t}=-f_{1} \phi_{2}(t)^{2}-2 f_{2} \phi_{1}(t)-2 f_{1} \phi_{1}(t) \phi_{3}(t),
\end{aligned}
$$

$\phi_{1}\left(t_{0}\right)=Q_{1}+Q_{2} \varepsilon+O\left(\varepsilon^{2}\right)$,

$\phi_{2}\left(t_{0}\right)=B_{1}+B_{2} \varepsilon+O\left(\varepsilon^{2}\right)$,

$\phi_{3}\left(t_{0}\right)=N_{1}+N_{2} \varepsilon+O\left(\varepsilon^{2}\right)$. 
$Q_{i}, B_{i}, N_{i}$ are the constants which are related to $C_{i}(i=1, \ldots, 14)$.

\section{CONCLUSION}

In summary, the AGCS method is successfully used to classify and construct approximate solutions of initial-value problem for Cahn-Hilliard equations which admit certain types of AGCSs. Therefore, it is interesting to study other types of nonlinear PDEs with perturbation term by AGCSs and we believe that some new results will be obtained.

\section{CONFLICT OF INTEREST}

The authors confirm that this article content has no conflict of interest.

\section{ACKNOWLEDGEMENTS}

This work has been Supported by NSFC (No.11326167, No.11226195, No.11401604), Natural Science Foundation of Henan Province (No.122300410166, No.142300410354), and Foundation of Henan Educational Committee (No.13A110119, 13A110117).

\section{REFERENCES}

[1] J.D. Cole, "Perturbation Methods in Applied Mathematics", Walthma: Blaisdell Publishing Company, 1968.

[2] A.H. Nayfeh, "Perturbation Methods", New York: John Wiley and Sons, 2000.

[3] G.W. Bluman and S. Kumei, "Symmetries and Differential Equations", New York: Springer, 1989.

[4] N.H. Ibragimov, "CRC Handbook of Lie Group Analysis of Differential Equations vol 3", Boca Raton, FL: Chemical Rubber Company, 1994.

[5] V.A. Baikov, R. K. Gazizov, and N. H. Ibragimov, and F. M. Mahomed, "Closed orbits and their stable Symmetries", J. Math. Phys., vol.35, no.1, pp.6525-6535, 1994.

[6] W.I. Fushchich and W. M. Shtelen, "On approximate symmetry and approximate solutions of the non-
[7] linear wave equation with a small parameter", J. Phys. A: Math. Gen., vol.22, no. 18, pp.L887-L890,1989.

[8] F.M. Mahomed and C. Z. Qu, "Approximate conditional symmetries for partial differential equations",J. Phys. A: Math. Gen., vol.33, no.2, pp.343-356, 2000

[9] S.L. Zhang, P. Z. Wang, and C. Z. Qu "Approximate Generalized conditional symmetries for the per-turbed general KdV-Burgers equations", Chin. Phys. Lett., vol.23, no.10, pp.2625-2628, 2006.

[10] X.Y. Jiao , R. X. Yao, and S. Y. Lou, "Approximate similarity reduction for singularly perturbed Boussi-nesq equation via symmetry perturbation and direct method", J. Math. Phys., vol.49, no.9, pp.093505 1-11, 2008.

[11] R.Z. Zhdanov , I. M. Tsyfra, and R. O. Popovych, "A precise definition of reduction of partial differentialEquations". J. Math. Anal. Appl., vol. 238, no.1,pp. 101-123, 1999.

[12] P. Basarab-Horwath and R. Z. Zhdanov, "Initial-value problems for evolutionary partial differential equa-tions and higher-order conditional symmetries", J. Math. Phys., vol.42, no.1, pp.376389, 2001.

[13] S.L. Zhang and J. N. Li, "Initial-value Problems for Extended KdV-Burgers Equations via GeneralizedConditional Symmetries", Chin. Phys. Lett., vol. 24, no.6, pp.1433-1436, 2007.

[14] W.I. Fushchych and R. Z. Zhdanov, "Conditional symmetry and anti-reduction of nonlinear heat equation", Proc. Acad. of Sci. Ukraine, no.5, pp. 40-44, 1994.

[15] A. Fokas and Q. Liu, "Generalized conditional symmetries and exact solutions of non-integrable equations", Theor. Math. Phys., vol.99, no.2, pp.571-582, 1994.

[16] J.N. Li and S. L. Zhang, "Approximate Symmetry Reduction for Initial-value Problems of the Extended KdV-Burgers Equations with Perturbation", Chin. Phys. Lett., vol. 28, no.3, pp.030201 14, 2011.

[17] J.W. Cahn and J. E. Hilliard, "Free energy of a nonuniform system. I. Interfacial free energy”, J. Chem.Phys., vol.28, no.2, pp.258267, 1958.

[18] S.M. Choo and S. K. Chung, "Asymtotic behaviour of the viscous Cahn-Hilliard equation”, J. Math.Comput., vol.11, no.1-2 pp.143-154, 2003.

[19] R. Rossi, "On two classes of generalized viscous Cahn-Hilliard equations", Commun. Pure Appl. Anal.,vol.4, no.2, pp.405$430,2005$.

[20] B. Stoth, "Convergence of the Cahn-CHilliard equation to the Mullins-Sekerka problem in sphericalSymmetry", J. Diff. Eqs., vol.125, no.1, pp.154-183, 1996.

[21] E.A. Carlen, M. C. Carvalho, and E. Orlandi, "A Simple Proof of Stability of Fronts for the Cahn-HilliardEquation",Commun Math. Phys., vol.224, no.1, pp.323-340, 2001. 